\title{
Astrovirus (HAstV) como agente causal de diarrea en niños Colombianos: Siete años de estudio
}

\author{
María Fernanda Gutiérrez PhD. ${ }^{1}$, Adriana Matiz MSc. ${ }^{1}$, Juan Carlos Ulloa MSc. 'Mónica Alvarado MI'. \\ 'Laboratorio de Virología, Grupo de Enfermedades Infecciosas, Departamento de Microbiología. Facultad de Ciencias. \\ Pontificia Universidad Javeriana. Bogotá, D.C. Colombia. \\ Correspondencia: mfgutier@javeriana.edu.co
}

Recibido: 26-05-2005 / Aceptado: 11-06-2005

\begin{abstract}
Resumen
Con el objeto de determinar el comportamiento de los Astrovirus como agentes causantes de diarrea en algunas regiones colombianas, se colectaron 1147 muestras de diarrea de niños menores de 5 años que acudieron a los servicios de consulta externa de cuatro centros de salud de regiones de Bogotá, Facatativa, Cartagena y Quibdo durante el periodo comprendido entre 1996 y el año 2002. La determinación de la presencia viral se realizó por medio de pruebas de ELISA encontrando una prevalencia global de 2,8\%. De las muestras positivas se seleccionaron 14 a las cuales se les obtuvo su ARN, se realizaron pruebas de RT-PCR y secuenciación de un segmento de 348 pb del ORF 2 del genoma viral para elaborar dendrogramas con el método de Neiborg joining y así, determinar su comportamiento filogenético. Las conclusiones generales de este estudio fueron:que en Colombia los HAstV poseen comportamientos endémicos y que las cepas colombianas no han sufrido cambios evolutivos que les permitan distanciarse de cepas de otros países.
\end{abstract}

Palabras claves: Diarrea, Astrovirus humano (HastV), RTPCR, filogenética.

\begin{abstract}
Astrovirus (HAstV) causing diarrea in colombian children: seven yeasr of study: In order to find the behavior of the Astrovirus isolates from diarrheic samples taken from children under 5 in four different cities of Colombian, there were tested 1147 samples between 1996 and 2002. The diagnosis of HastV was done by EIA and there were found $2,8 \%$ of global prevalence. Fourteen of the positive samples were typed by RT PCR and sequencing the $384 \mathrm{bp}$ region of the ORF2 segment of the viral genome (which codes for the capsid protein). Neighbor joining was done in order to make the phylogenetic study. The conclusions of the study were that in Colombia, HastV has an endemic behavior and the Colombian strains, obtained from samples of geographically distant or the same places, show that there is no genetic variability among the same genotype and among strains with the same typed but collected out of this country.
\end{abstract}

Key words: Diarrhea, Human Astrovirus (HastV), RTPCR, phylogenetic.

\section{Introducción}

Los Astrovirus, son virus ARN de cadena sencilla, sin envoltura que fueron descritos por primera vez en
1975 y han sido reportados como causantes del 2 al $8 \%$ de las diarreas de etiología viral (EDA) en el mundo. La mayoría de estudios epidemiológicos se han 
realizado en países desarrollados y con estaciones donde este virus presenta un comportamiento epidémico asociado a épocas de invierno. Dentro de las características epidemiológicas importantes están el que afecta principalmente niños menores de dos años, tiene amplia distribución mundial y pueden presentarse solos o coinfectando con otros virus entéricos (1-5).

Los Astrovirus humanos (HAstV) han sido clasificados en 8 tipos antigénicos denominados HAstV 1 a HAstV 8 de los cuales el tipo predominante reportado es el HAstV 1. En países en vías de desarrollo y climas sin estaciones definidas como el nuestro, existen pocos reportes epidemiológicos, en Perú se presenta el tipo 1 como predominante, seguido por el 2 y el 4, de manera muy similar a Santiago de Chile donde además del tipo 4 apareció también el tipo 3 (2, 4, 6). En cuanto a la edad de la población afectada, se sabe que este virus ataca a menores de 5 años aunque se ha reportado en ancianos y personas con deficiencias inmunes. En Francia, Australia y Alemania se observó una mayor prevalencia en niños menores de los 7 meses (1-3).

El genoma de los HAstV esta compuesto por 6.813 nucleótidos (nt) organizados en tres ORFs, donde el ORF1a codifica para una proteasa, el ORF1b para la polimerasa y el ORF2 localizado en el extremo 3' del genoma, se sobrepone con el ORF 1b, codifica para la proteína precursora de la cápside y se presenta adicionalmente como RNA subgenómico.

Debido a la poca inmunogenicidad de la proteína de la cápside, ha sido difícil producir anticuerpos monoclonales útiles en técnicas diagnósticas y de serotipificación, y es por eso que la clasificación viral está basada en el genotipo que se asocia de manera clara con el serotipo (7-10).

Dentro del ORF 2 a nivel de los nt 258 a 606, existe una región de 348 pb que corresponde al 15\% del total del ORF 2 y que ha sido comúnmente utilizada para los estudios de clasificación viral. Esta región se amplifica con la técnica de RT-PCR con los amplímeros Mon 244 y Mon 245 que fueron utiliza- dos en este trabajo en donde además se realizó una secuenciación y se dio paso a los estudios de similitud filogenética (4).

Conocer el comportamiento epidemiológico de un virus en una población es el primer paso para elaborar estrategias de prevención y control. El objetivo de este trabajo fue reunir los resultados que con respecto a HAstV se han realizado en los últimos años en el país y presentar un reporte del comportamiento de este virus que ha sido poco valorado debido al proceso autolimitado de la infección que causa y a la baja prevalencia reportada en otros países

\section{Materiales y métodos}

Muestras: A partir de 1996 se han recolectado un total de 1147 muestras diarreicas de niños menores de 5 años que ingresaron por consulta externa a centros asistenciales de las ciudades de Bogotá, Cartagena, Quibdo y Facatativa. Las muestras fueron recolectadas en los laboratorios de la región donde además se les realizó examen coprológico y coproscópico.

Los requisitos de inclusión para las muestras al estudio fueron: que pertenecieran a niño menor de cinco años, que presentaran diarrea (evacuación de treo o mas deposiciones acuosas o líquidas), que la cantidad de muestra fuera suficiente para realizarle la determinación de este y otros agentes patógenos y que fuera llenado un formato con sus datos y el consentimiento informado donde la madre o acudiente diera permiso para realizarle a la materia fecal del niño algunas pruebas distintas al coproscópico

Una vez recolectada la muestra, fue alicuotada, enviada al laboratorio de Virología de la Universidad Javeriana y almacenada a $-20^{\circ} \mathrm{C}$ hasta su utilización.

Determinación de la presencia de Astrovirus por ELISA: Las 1147 muestras fueron procesadas de acuerdo a las instrucciones del estuche comercial (DAKO USA ) el cual tiene fijo a su fase sólida un anticuerpo que captura la partícula viral presente en la materia fecal. La reacción antígeno anticuerpo es 
detectada mediante un segundo anticuerpo dirigido contra el virus y conjugado a peroxidasa cuya presencia es revelada con su correspondiente sustrato. La lectura se realizó en un equipo Multiskan MCC/340

Tipificación viral: Para confirmar la presencia viral y obtener el segmento a secuenciar, los ARNs de las muestras positivas por ELISA, fueron extraídos a partir $20 \%$ de materia fecal diluida en PBS $1 \mathrm{X}$ mediante los sistemas de Trizol R (GIBCO) y QIA Amp Viral Kit (Qiagen, Hilden, Germany). La amplificación por RT-PCR se realizó con los amplímeros Mon244 y Mon 245, utilizando el protocolo descrito por Noel y col [4]. La genotipificación fue hecha directamente de la secuencia del producto de PCR con el método ABI Prism Big Dye Terminator Cycle sequencing Ready Reaction kit (perkin-Elmer Biosystems) en un secuenciador automático (modelo $373^{\text {a }}$ DNA Sequencing System, PE Biosystems) y se realizó en los laboratorios de Virología CMBC, IVIC en Venezuela, en el laboratorio de Virología y Servicio de Pediatría, Hospital de Niños, Facultad de Medicina y Farmacia, Dijon, Paris, Francia y en el laboratorio de Virología del Departamento de Microbiología, Instituto de ciencias biomédicas de la Universidad de San Pablo, Brasil.
Análisis del comportamiento filogenético de las cepas colombianas: Las secuencias obtenidas fueron alineadas con el programa FASTA (www.infobiogen.fr) y comparadas con las secuencias de cepas previamente reportadas en el Genbank, adicionalmente se estudio su similaridad mediante el programa ClustalW [11]. Empleando el programa Mega 3.0 se obtuvo una matriz binaria que fue analizada por el método filogenético Neighbor joining (NJ) para estimar la filogenia generandose dendrogramas a los que se les aplicó un soporte (bootstrap) de 550 repeticiones. De esta manera se logró visualizar el comportamiento de las cepas colombianas entre si y se compararon con algunas cepas extranjeras escogidas al azar utilizadas como referencia.

\section{Resultados}

Entre Abril de 1996 y Mayo del 2002 fueron recolectadas 1147 muestras diarreicas de niños menores de 5 años que consultaron por EDA al servicio unidades de consulta externa de 4 regiones colombianas. A la totalidad de las muestras se les determinó la presencia de HAstV por ELISA encontrándose una prevalencia 2.8\% (Tabla 1). Bogotá fue la región donde mas HAstV se encontró con un $4.7 \%$ de prevalencia y Quibdo la de menor prevalencia con un $1.3 \%$.

Tabla 1. Distribución de los HAstV en Colombia desde 1996 hasta el 2002. Por edad y por sexo: Distribución de las muestras positivas de acuerdo a rangos de edad distribuida por meses (0-3, 4-6, 7-12, 13-24, 25-36, 37-48 y 49-60 meses).

\begin{tabular}{|l|c|c|c|c|c|c|c|}
\hline \multicolumn{1}{|c|}{ Ciudad } & Fecha & $\mathbf{n}$ & Positivas & $\%$ & $\begin{array}{c}\text { Por edad } \\
\text { (meses) }\end{array}$ & $\begin{array}{c}\text { Por sexo } \\
\text { Masc/Fem \% }\end{array}$ & $\begin{array}{c}\text { Cepas } \\
\text { tipificadas }\end{array}$ \\
\hline Bogotá & $1996-99$ & 251 & 11 & 4.7 & $7-48$ & $41 / 58$ & 8 \\
\hline Facatativa & $1999-00$ & 300 & 8 & 2.7 & $0-48$ & $62.5 / 37.5$ & 5 \\
\hline Cartagena & $2001-03$ & 137 & 4 & 2.9 & $7-12$ & $50 / 50$ & 1 \\
\hline Quibdo & $2001-03$ & 221 & 3 & 1.3 & $0-36$ & $66 / 33$ & \\
\hline Facatativa & $2001-03$ & 238 & 6 & 2.5 & $0-48$ & $33 / 66$ & \\
\hline \multicolumn{1}{|c|}{ TOTAL } & & 1147 & $\mathbf{3 2}$ & $\mathbf{2 . 8 2}$ & & $\mathbf{5 0 . 5 / 4 9 . 5}$ & 14 \\
\hline \hline
\end{tabular}

n: número de muestras recolectadas. \%: Porcentaje de muestras positivas para HAstV . 
Tabla 2: Registro de las 14 cepas secuenciadas y tipificadas en Colombia. Tipo: serotipo viral. Fecha: Año de recolección de la muestra. Lugar: Ciudad o Municipio donde se recolectó la muestra.

\begin{tabular}{|c|c|c|c|c|c|c|c|c|c|}
\hline Nombre & tipo & $\begin{array}{l}\text { Número en } \\
\text { Genbank }\end{array}$ & Fecha & Lugar & Nombre & Tipo & $\begin{array}{c}\text { Número en } \\
\text { Genbank }\end{array}$ & Fecha & Lugar \\
\hline COL509 & 1 & AF211958 & \multirow{8}{*}{1996} & \multirow{8}{*}{ Bogotá } & 113 & 4 & DQ157435 & \multirow{5}{*}{1999} & \multirow{5}{*}{ Facatativa } \\
\hline COL526 & 1 & AF211959 & & & 114 & 4 & DQ157436 & & \\
\hline COL505 & 3 & AF211960 & & & 115 & 4 & DQ157437 & & \\
\hline COL664 & 2 & AF211961 & & & 116 & 5 & DQ157439 & & \\
\hline COL546 & 2 & AF211962 & & & 117 & 5 & DQ157440 & & \\
\hline COL673 & 1 & AF211963 & & & 359 & 1 & DQ157438 & 2002 & Cartagena \\
\hline COL503 & 1 & AF211956 & & & & & & & \\
\hline COL418 & 1 & AF211957 & & & & & & & \\
\hline
\end{tabular}

Al realizar el estudio descriptivo para determinar en que edad y si HAstV aparecía en niños de sexo masculino o femenino, se observó que la infección prevalece en niños entre los 7 y los 48 meses (con mayor prevalencia entre 7 y 24 meses) sin que esto haya tenido un resultado estadísticamente significativo (valores de $\mathrm{p}$ mayores a 0,05$)$. Con respecto al género al cual pertenecen los niños, se observó que da de igual manera en niños de género masculino que femenino. La tabla 2 se realizó para determinar la procedencia y el año en el cual se obtuvo cada cepa y relacionarlo con el resultado del comportamiento filogenético por NJ.
Del número total de muestras positivas, sólo 14 pudieron ser tipificadas obteniéndose 5 cepas serotipo 1, 3 muestras serotipo 2, 1 muestra serotipo 3, 3 muestras serotipo 4 y 2 muestras serotipo 5. Para encontrar las similitudes filogenéticas se realizaron dos árboles mediante el método algorítmico de $\mathrm{NJ}$, los cuales aparecen en las figuras 1 y 2. La figura 1 muestra el comportamiento de las cepas Colombianas con sus correspondientes serotipos y en la figura 2 se incluyó una cepa externa escogida al azar con el objeto de evidenciar su capacidad de apareamiento con las cepas colombianas.

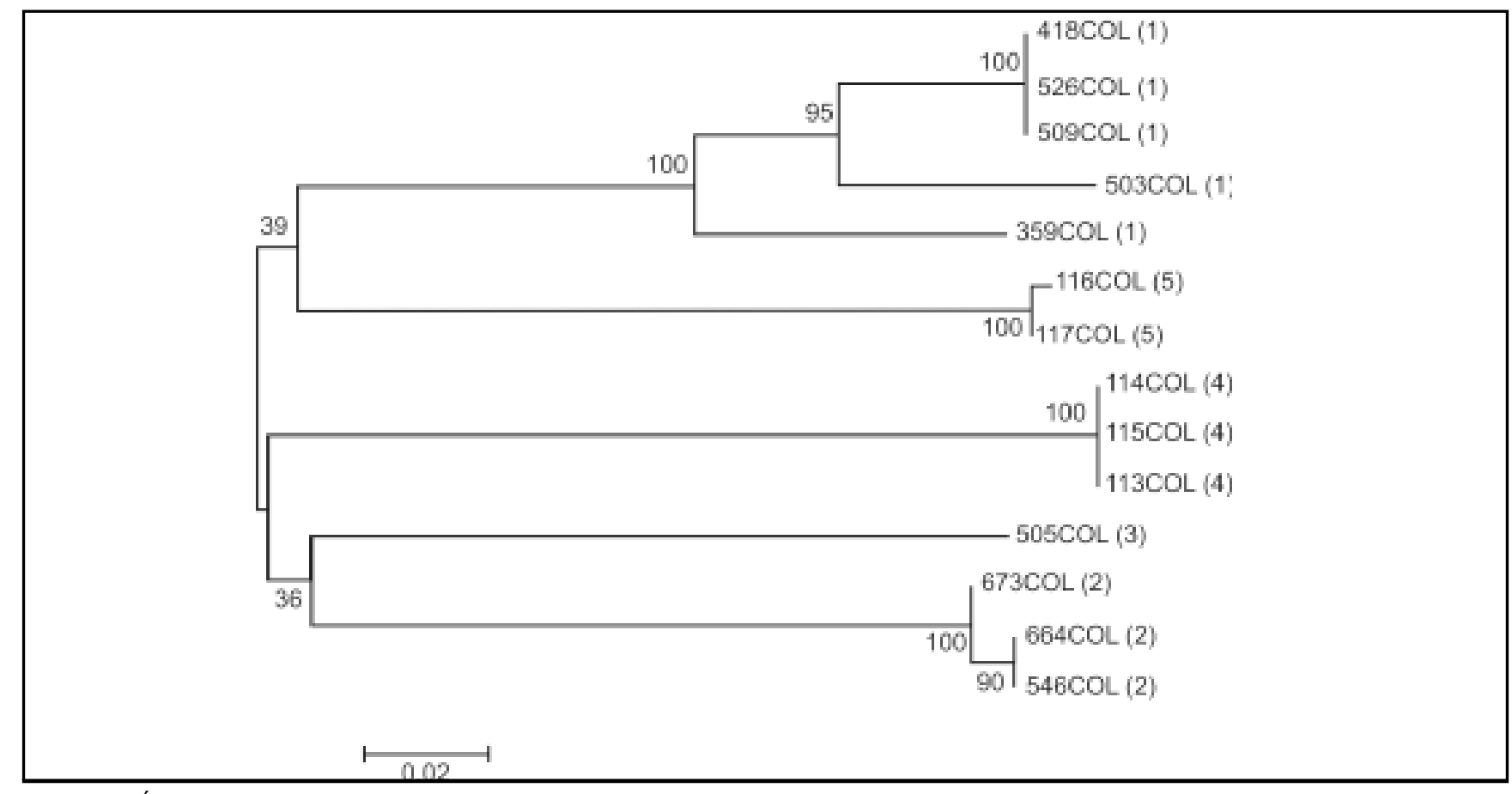

Figura 1. Árbol filogenético de HAstV aislados de pacientes colombianos, obtenidos desde 1996. Elaborado con el método NJ, con "bootstrap" de 550 repeticiones. 


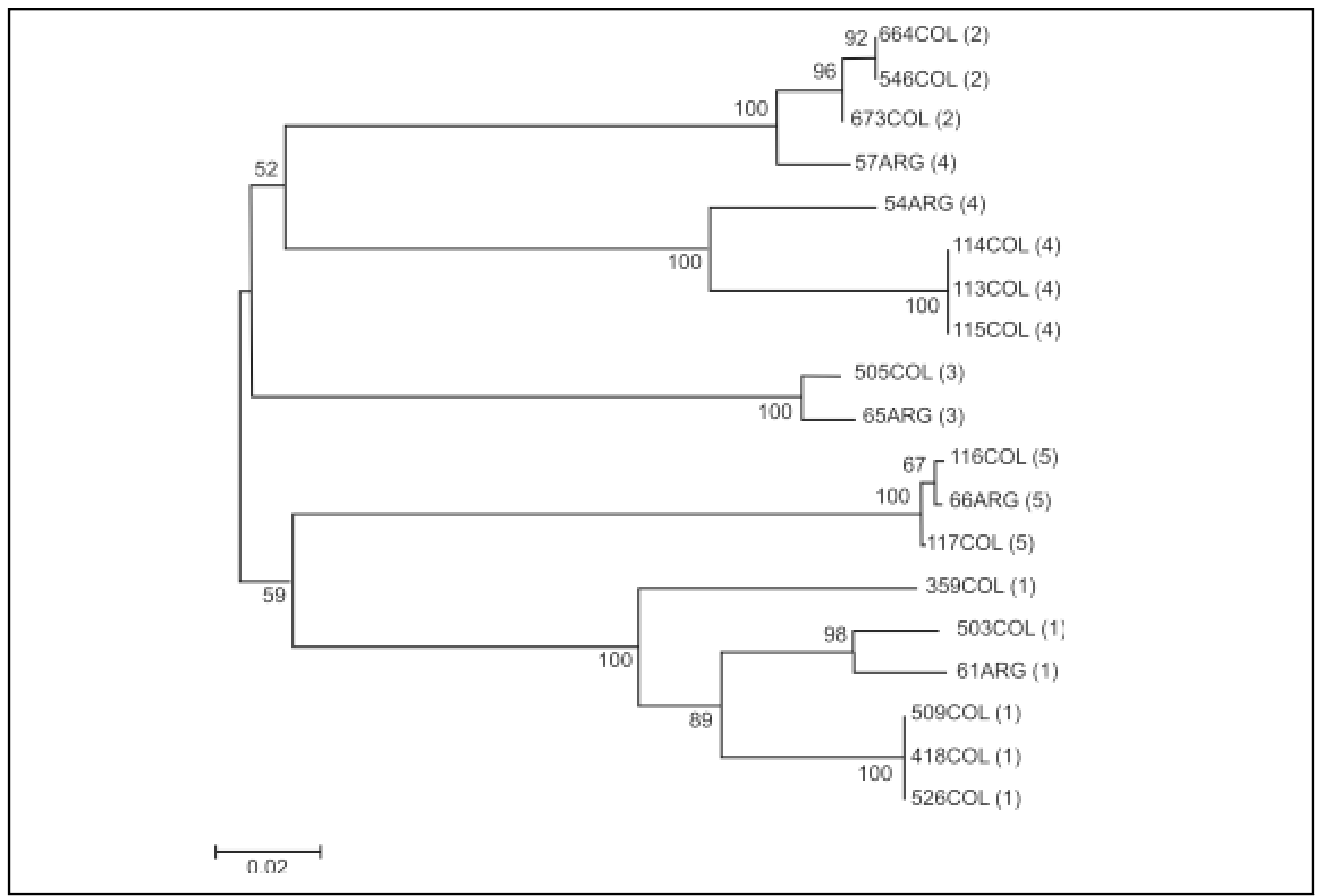

Figura 2. Árbol filogenético de HAstV aislados de pacientes colombianos y de cepas de referencia de HAstV aislados fuera de Colombia obtenidos desde 1996. Elaborado con el método NJ, con "bootstrap" de 550 repeticiones.

\section{Discusión}

Los HAstV son virus causantes de diarrea, muy poco estudiados en Colombia por su bajo impacto en pediatría. Al tratar de dilucidar las causas de las diarreas en países en vías de desarrollo como Colombia se deben buscar primero los Rotavirus del grupo A, en segundo lugar los Norovirus que han sido reportados como causantes de endémias y por último, (12) otras especies virales como los HAstV y los Adenovirus entéricos. Cuando se evalúan los HAstV, se detectan prevalencias muy bajas que no alcanzan a elevar el porcentaje de las diarreas diagnosticadas en un tipo de poblaciones como la nuestra (13-17).

En este estudio se ha demostrado que las prevalencias mundiales son las mismas encontradas en Colombia, no importando la zona geográfica de donde provenga el menor. Un $2,8 \%$ de prevalencia a nivel nacional demuestra que este porcentaje se acerca a la prevalencia mas baja reportada en los países desarrollados que es del $2 \%(18,19)$.

Con respecto a la edad, se sabe que la lactancia es una época de inmunidad a muchos agentes patógenos debido a los anticuerpos maternos obtenidos de la madre (20). Una vez terminada esta etapa, el infante inicia el proceso de reacción a los agentes patógenos, sin mucho éxito protectivo, lo que aumenta la posibilidad de infección por HAstV, como lo demuestran los resultados descritos en la tabla 1. El sistema inmune pasa unos años de "ataque" por varios patógenos donde los niños (entre 7 meses y 3 años) tienden a infectarse mas que los niños en edad escolar.

Algunos autores han propuesto que los niños son más propensos que las niñas a la contaminación por HAstV; sin embargo en este estudio no se encontraron diferencias significativas en relación al género o al estrato 
socioeconómico, indicando que estas variables no influyen en el desarrollo EDA de etiología viral (21).

Los HAstV tipo 1 han sido los mas frecuentemente reportados causando EDA (7), sin embargo, los resultados obtenidos en Colombia muestran que los tipos del 1 al 5 aparecen y desaparecen si mantener un comportamiento epidemiológico predecible o cíclico. En la tabla 2 se observa que el serotipo 1 prevaleció entre los años 1996 al 1999 lo cual cambió de tal manera que en el año 1999 desapareció y aparecieron los serotipos 4 y el 5 (ausentes en los años anteriores). Para el año 2002, reaparece el serotipo 1 pero al observar su comportamiento en el árbol filogenético, se aprecia una distancia filogenética que es proporcional al número de mutaciones que ha sufrido el ancestro, separándose genéticamente de las cepas reportadas en el siglo anterior. Esta variabilidad viral ha sido descrita por varios autores quienes consideran que la ausencia de mecanismos reparadores en la síntesis de RNA por falta de una RNA polimerasa reparadora, es el motivo de hipermutaciones, unos de los mecanismos evolutivos responsables de estas variaciones y de la aparición de nuevas cepas o serotipos (22).

La tarea de la filogenia molecular es convertir información de secuencias en árboles que demuestren evolución. NJ es in método de inferencia utilizado para formar grupos basado en algoritmos matemáticos que revierten la información en relaciones de similaridad entre las cepas

En la figura 1 se observa como los HAstV colombianos de cada serotipo se agrupan de manera conservada. Las cepas 418, 526 y 509 poseen secuencias de nt idénticas mientras que la cepa 503 , recolectada en el mismo período de tiempo, posee alteraciones en la secuencia nucleotídica que genera la apertura de la rama. La cepa 359 fue recolectada varios años después y en lugar distante (Cartagena) y presenta varias mutaciones que generan una mayor apertura del árbol, mostrando divergencia entre las cepas serotipo 1 de HAstV en Colombia. Algunos autores han propuesto que la variación en esta cepa alcanza una distancia filogenética que justificaría la aparición de un subtipo viral, lo que aún está en estudio.

Las tres cepas del serotipo 4 fueron recolectadas en la misma región y en el mismo periodo de tiempo y por su comportamiento filogenético podría pensarse que es la misma cepa. El comportamiento de los serotipos 2 y 5 fue bastante similar al 4; en relación a la cepa del serotipo 3 no se encontró otra muestra para realizar comparación.

La figura 2 trata de completar el conocimiento anterior comparando las cepas colombianas con cepas de referencia extranjeras obtenidas del Genbank. Los resultados en los casos de los serotipos 2, 4 y 3 muestran que en otros países, existe un número mayor de variaciones, lo cual no sucedió con las cepas de los serotipos 1 y 5 ; grupos colombianos en los cuales las cepas extranjeras quedaron inmersas.

\section{Conclusiones}

En Colombia los HAstV no poseen comportamientos epidemiológicos distintos a los reportados en otros países lo cual no significa que puedan ser excluidos cuando se buscan los agentes etiológicos de la EDA. Por otro lado se demostró que las cepas colombianas no han sufrido cambios evolutivos que les permitan distanciarse de cepas de otros países lo que permite inferir que si bien no existen enzimas reparadoras que mantengan la estabilidad de la secuencia de nucleótidos, el genoma viral se mantiene muy estable tanto con el paso del tiempo como con el cambio de lugar.

Agradecimientos: Al Dr. Juan E. Ludert del laboratorio de Virología CMBC, IVIC en Venezuela, la Dra. Fabianne Bon del laboratorio de Virología y Servicio de Pediatría, Hospital de Niños, Facultad de Medicina y Farmacia, Dijon, Paris, Francia y la Dra. Maria Lucia Racz del laboratorio de Virología del departamento de Microbiología, Instituto de Ciencias Biomédicas de la Universidad de San Pablo, Brasil. Por último a las estudiantes de bacteriología de la Universidad Javeriana, con quien se realizó el muestreo y la detección viral. 


\section{Referencias}

1. Bon F, Fascia P, Dauvergne M, Tenenbaum D, Planson H, Petion A, Porthier P, Kohli E. Prevalence of group A Rotavirus, Human Calicivirus, Astrovirus, and Adenovidrs type 40 and 41 infections among children with acute gastroenteritis in Dijon, France. The J Clin Microbiol. 1999; 37(9): 3055-3058.

2. Gaggero A, O'Ryan M, Noel J, Glass R, Monroe S, Manani N, Prado V, Avendaño L. Prevalence of Astrovirus Infection among Chilean children with acute gastroenteritis. The J Clin Microbiol. 1998; 36(12): 3691-3693.

3. Schulz K, Wegner U, Gurtler L, Wiersbitzky S, Mentel R. Analysis of genotypes of Human Astrovirus isolates from hospitalized children in Northaeasten Germany. Eur J Clin Microbiolo Infect Dis. 2000; 19:563-565.

4. Noel, J.S., Lee, T.W., Kurtz, J.B., Glass, R.I., Monroe, S.S., Typing of Human Astrovirus from clinical isolates by Enzyme immunoassay and nucleotide sequencing. The J Clin Microbiol, 1995. 33(4): p. 797-801.

5. Mitchell D, Matson D, Jiang K, Berke T, Monroe S, Carter M, Willcocks M, Pickering L. Molecular Epidemiology of childhood Astrovirus Infection in child care centers. The J Infect Dis. 1999; 180: 514-517.

6. Medina. S.M., G., M.F., Liprandi, F., Ludert, J.E., Identification and type distribution of Astrovirus among children withn gastroenteritis in Colombia and Venezuela. The J Clin Microbiol. 2000; 38(9): p. 3481-3483.

7. Krishna N. Identification of structural domains involved in Astrovirus capsid biology. Vir Immunol. 2005; 18(1):17-26.

8. McKee L. Ethnomedical treatment of children's diarrheal illness in the highlands of Ecuador. Soc Sci Med. 1987; 25(10):1147-1155

9. Marczinke B, Bloys A, Brown D, Willcocks M, Carter M, Brierley I. The human Astrovirus RNA-dependent RNA polymerase coding region is expressed by ribosomal frameshifting. J Virol. 1994; 68(9): 5588-5595.

10. Palombo E, Bishop R. Annual incidence, serotype distribution and genetic diversity of human Astrovirus isolates from hospitalized children in Melbourne, Australiz. The J Clin Microbiol. 1996; 34(7): 1750-1753.

11. Higgins D, Thompson J, Gibson T, Thompson J, Higgins D, Gibson T, CLUSTAL W: Improving the sensitivity of progressivemultiple sequence alignment through sequence weighting,position-specific gap penalties and weight matrix choice. Nuc Acid Res. 1994; 22: 4673-4680.
12. Martínez L, Matiz A, Trespalacios A, Ajami N, Mora C, Serrano P, Mercado M, Gutiérrez M. Etiología de la enfermedad diarreica aguda en niños menores de 5 años en la población de Quibdo. El Calicivirus, un nuevo hallazgo. Pediat. 2005; 40(1): 43-52.

13. Giordano M, Ferreyra L, Martinez L, Yudowsky S, Nates S. The epidemiology of acute viral gastroenteritis in hospitalized children in Cordoba, Argentina: an insight of disease burden. Rev Inst Med Trop San Paulo. 2001; 43(3):193-197.

14. Guix S, Caballero S, Villena C, Bartolomé R, Latorre C, Rabella N, Simó M, Bosch A, Pintó R. Molecular epidemiology of Astrovirus infection in Barcelona, Spain. The J Clin Microbiol. 2002; 40(1):133-139.

Guerrero M, Noel J, Mitchell D, Calva, J. Epidemiologia de Astrovirus en la ciudad de México. Enfer Infec Microbiol. 1999;19(2):106-108.

15. Mustafa H, Palombo E, Bishop R. Epidemiology of Astrovirus Infection in Young children hospitalized with acute gastroenteritis in Melbourne, Australia, over a period of four consecutive years, 1995 to 1998 . The J Clin Microbiol. $2000 ; 38(3): 1058-1062$

16. Utagawa E, Nishizawa S, Sekine S. Astrovirus as a Cause of Gastroenteritis in Japan. The J Clin Microbiol. 1994; $32: 1841-1845$.

17. Grimwood K, Carzino R, Barnes G, Bishop R. Patients with enteric Adenovirus gastroenteritis admitted to an Australian Pediatric teaching hospital from 1981 to 1992 . The J Clin Microbiol. 1995; 33(1):131-136.

18. Harsi, C.M.R., D.P.; Gomes, S.A.; Gilio, A.E.; Stewien, K.E.; Baldacci, E.R. and Candeias, J.A.N., Adenovirus genoma types isolated from stools of children with gastroenteritis in Sao Paulo, Brazil. J Med Virol. 1995; 45:127-134.

19. Cukor G, Blaclow N. Human Viral Gastroenteritis. Microbiol Rev. 1984; 48(2): 157-199.

20. Gutiérrez M, Mercado M, Matiz A, Parra M, Riaño M. Características epidemiológicas y moleculares de los agentes causantes de diarrea infantil en una población colombiana. Premio a la Investigación 2004 Biosystem, 2004.

21. Belkum A, Struelens M, Visser A, Verbrugh H, Tibayrenc M. Role of genomic typing in taxonomy, evolutionary genetics and microbial epidemiology. Clin Microbiol Rev. 2001;14(3): 547-560 